\title{
Modeling Moisture Redistribution of Pulse Drip Irrigation Systems by Soil and System Parameters: New Regression-based Approaches
}

Bakhtiar Karimi ( $\sim$ bakhtiar.karimi@gmail.com )

University of Kurdistan

Nazir Karimi

University of Kurdistan

Jalal Shiri

University of Tabriz

Hadi Sanikhani

University of Kurdistan

\section{Research Article}

Keywords: Drip irrigation, Irrigation management, Modeling, Moisture front, Redistribution

Posted Date: March 1st, 2021

DOl: https://doi.org/10.21203/rs.3.rs-267556/v1

License: (9) This work is licensed under a Creative Commons Attribution 4.0 International License. Read Full License 

system parameters: new regression-based approaches

\section{Abstract}

One of the strategies for increasing water use efficiency and reducing deep percolation drip irrigation systems is considering the patterns of moisture redistribution after cut-offing the irrigation process. An experimental study was conducted in the present research to evaluate the moisture redistribution process under surface and subsurface pulse drip irrigation systems and developing new regressionbased methodologies for estimating moisture redistribution dimensions using both the soil and system parameters together. A physical model was made and the experiments were performed on three different types of soil texture (light, medium, and heavy) with three emitter flow rates $(2,4$, and 6 lit/hr) in three emitter installation depths $(0,15$, and $30 \mathrm{~cm})$. The experiments were conducted for both continuous (CI) and pulse (PI) irrigation modes. The results showed that significant amounts of wetting dimensions and wetted area of the moisture bulb are related to post-cut-offing stage. Then, using the nonlinear regression analysis, several models were proposed to estimate the 
emitter). The comparison of the measured and the stimulated values indicated that the non-linear regression models simulated the parameters associated with redistribution, accurately.

Keywords: Drip irrigation, Irrigation management, Modeling, Moisture front, Redistribution

Highlights:

- Moisture re-distribution dimensions under drip irrigation systems were simulated.

- Experiments were conducted for both surface and sub-surface systems.

- Nonlinear regression models were established and proposed for simulations.

\section{Introduction}

Drip irrigation systems are among the suitable and efficient irrigation methods due to their high water use efficiency and yield production, especially in arid and semi-arid regions, where the fresh water is a limited source (Liu and $\mathrm{Xu}, 2018$; Karimi et al., 2020). Under suitable designing and implementation procedures, drip irrigation systems usually provide higher irrigation efficiency values than the traditional surface irrigation systems (Qiaosheng et al., 2007; Al-Ogaidi et al., 2016). Appropriate design and implantation of drip irrigation systems depends on an accurate understanding of the wetting patterns (e.g. Yao et al., 2011; Kandelous and Simunek, 2010b; Elmaloglou and Diamantopoulos, 2010; Hammami and Zayani, 2016; ). The pattern of moisture distribution is a function of soil physical characteristics, distance of emitters and laterals, emitter outflow rate, emitter installation depth, and water application mode (continuous or pulse) (Singh et al., 2006 Al-Ogaidi et al., 2016; Khattak et al. 2017; Malek and Peters, 2011; Golestani Kermani et al., 2019). As mentioned, two irrigation models might be followed by using drip irrigation systems, 
namely, the continuous and pule irrigation modes (in brief, CI and PI, respectively). The pulse mode consists of a set of cycles; each of them is constituted from an irrigation phase and a resting phase. Substantial studies have been carried out on the pulse irrigation mode so far, confirming that the patterns of moisture distribution in this mode is substantially different than the continuous irrigation mode (e.g. Karmeli and Peri, 1974; Levin et al., 1979; Mostaghimi and Mitchell, 1983; Mohammad

Beigi et al., 2017). Accurate simulation of the wetting dimensions can reduce the applied water volume. It also provides suitable knowledge for choosing the suitable distance between the laterals and emitters. There are mainly three categorizes of studies dealing with simulating the moisture distribution issues, namely, experimental (e.g. Schwartzman and Zur, 1986; 2006; Qiaosheng et al., 2007), numerical (e.g. Elmaloglou et al., 2013; Arbat et al., 2013; Šejna et al., 2014) and analytical solutions (e.g. Cook et al., 2003; Hammami and Zayani, 2016). Meanwhile, numerous studies have demonstrated that the empirical models are easier and simpler than the analytical and numerical methods, comprising lower computational complexity. Moreover, analytical and numerical approaches need considerable computational cost and higher skills; so it is impossible to employ them for design purposes (Malek and Peters, 2011; Shiri et al. 2020). One of the important parameters for accurately determining the moisture dimensions in the drip irrigation systems is wetting redistribution, which usually occurs after cut-offing the irrigation process in both the horizontal and vertical directions. Considering moisture redistribution values can reduce the wetting front overlapping between the laterals and emitters (in case of horizontal redistribution) as well as water deep percolation (in case of vertical redistribution), which can totally reduce the applied water volume (Mohammad Beigi et al., 2017). Moisture redistribution may depend on various soil or irrigation system parameters that make its quantification difficult in practical issues. According to Karimi et al. (2013), moisture redistribution is a function of soil properties, outlet emitter flow rate and emitter installation depth. Mohammadbeigi et al. (2016), on the other hand, introduced the irrigation mode (continuous/pulse water flow) as an additional affecting factor that considerably 
change the redistribution patterns. Based on their results, significant amounts of the existing moisture distribution are associated with redistribution (i.e., in the clay soils with flow rate of 2.4 lit/hr, $23 \%$ of the vertical distribution of soil moisture is associated with redistribution). Their results also showed that the moisture redistribution in the continuous irrigation system was $4-7 \%$ more than the pulse irrigation. Summarizing, the important factors of soil moisture redistribution are horizontal redistribution $\left(\mathrm{R}_{\mathrm{re}}\right)$, downward vertical redistribution $\left(\mathrm{V}_{\mathrm{re}}\right)$, upward vertical redistribution $\left(\mathrm{V}_{\text {re-up }}\right)$, the lower wetted area $\left(\mathrm{A}_{\mathrm{re}}\right)$ and the upper wetted area $\left(\mathrm{A}_{\mathrm{re}-\mathrm{up}}\right)$ after cut-offing the irrigation process. These parameters are represented in Fig. 1. In the figure, $A_{\text {irr }}$ is the total wetted area at the time of irrigation.

So far, several studies have been conducted to establish relations between the moisture redistribution dimensions and their affecting parameters. Among others, Karimi et al. (2015b) presented some relations for estimating horizontal and vertical redistribution pattern of the moisture front in the continuous irrigation system, using dimensional analysis (Buckingham $\pi$ theorem). Elmaloglou and Diamantopoulos (2009) investigated the effect of hysteresis phenomenon on soil moisture redistribution and deep percolation losses in pulse and continuous irrigation systems and concluded that deep percolation loss (with and without the hysteresis phenomenon) has been considerably decreased in pulse irrigation system. The previous studies showed that there are few studies on simulating soil moisture redistribution pattern, especially for subsurface irrigation systems, while numerous studies have been carried out to estimate the wetted dimensions of moisture bulb under surface drip irrigation system. Further, most of the previous studies have focused on either moisture redistribution modeling for the continuous irrigation mode or simulating wetted area of moisture bulb after cut-offing the irrigation process. Nevertheless, analysis of moisture redistribution is an important task in both surface and subsurface irrigation systems for determining the lateral/emitter distance and emitter installation depth, which might be carried out through regression-based techniques. Therefore, one of the main objectives of this study was to investigate/simulate the 
horizontal and vertical redistribution patterns for different soils and outflow rates under continuous and pulse surface/subsurface irrigation systems.

\section{Material and method}

The experiments were performed between April 3, 2016 and October 12, 2017. A rectangular cube model with dimensions of $3 * 1 * 0.5 \mathrm{~m}$ was constructed at the University of Kurdistan. The front cube was split into three equal parts and three experiments were conducted simultaneously. To prevent the preferential flow during the experiments, a relatively rough surface was created by applying glue on the surface of the flat Polycarbonate and outpouring the coarse gravels on it, so that the transparency of the front page of the model was preserved (Kandelous and Simunek, 2010a). All equipment and facilities of an irrigation system were simulated in accordance with actual field conditions in the physical model. Water flow was delivered from a 200-liter tank to the emitters (Netafim emitters) by means of polyethylene pipes (i.e., the main pipe with a diameter of $32 \mathrm{~mm}$, and sub-main and lateral pipes with diameters of 20 and $16 \mathrm{~mm}$, respectively). Additionally, in the route of water conveyance from the valve, a screen filter (to prevent emitter clogging and distribution non-uniformity), a pressure gauge (to maintain a constant pressure of 2 bars in all experiments), and a flow on-off valve (to control flow into each compartment) were installed. Considering that the amount of outflow rate was very low, a bypass collection was also designed to reduce the pressure exerted to the system (Karimi et al., 2015a). As the experiments have been done on a hemisphere, the value of the outflow rate must be multiplied by 2 in concordance to the actual conditions (Li et al., 2003, Kandelous and Simunek, 2010, Al-Ogaidi et al., 2016). Fig. 2 shows the full view of the position of all the equipment used in the study. Table1 presents the physical characteristics of the studied soil textures. The saturated hydraulic conductivity was estimated using Rosetta software (Schaap et al., 2001). Filling each compartment by dried soil was carried out on the basis of the soils bulk density. In order to stabilize the soil and uniformly distributing the initial 
moisture, the soil inside the compartments were placed in the laboratory for 24 to $48 \mathrm{~h}$ (Al-Ogaidi et al., 2016). Variations of the initial moisture content in different treatments were low in accordance to literature (e.g. Shiri et al., 2020; Al-Ogaidi et al., 2016). Finally, after completing the irrigation process, the redistribution wetting front was recorded for different times (e.g., 3, 6, 18, 42, and 66 h) on the Polycarbonate page. Total irrigation time for continuous, 40-20, 30-30 and 20-40 treatments

were 4, 12, 8 and 6 hours, respectively. However, the active time (on-time) for all treatments was 4 hours that made the volume of applied water for all treatments equal. At the end of each experiment, by preparing a photograph of the front page of the model and using the Grapher software, the redistribution values were calculated in the horizontal and vertical directions.

The experiments were performed for three kinds of soil textures (coarse, medium and fine), three types of emitter flow rates $(2,4$, and 6 lit/hr), three different emitter installation depths $(0,15$, and 30 $\mathrm{cm}$ ) and four kinds of irrigation modes (CI, PI 30-30, PI 20-40, and PI 40-20 minutes; where the first number refers to the irrigation time and the later shows the resting (off) time of the system in each cycle), thus combining 108 kinds of experimental treatments. .

\section{Descriptions of the suggested models}

Recently, several studies have been carried out for simulation of wetting front dimensions using nonlinear regression technique (e.g. Al-Ogaidi et al., 2016; Malek and Peters, 2011). By using this method, the wetting front position can be simulated through considering more input variables and appropriate understanding of the complex soil environment. As mentioned, different factors may affect the pattern of soil moisture redistribution, from which, some are related to soil physical characteristics, such as saturated hydraulic conductivity, initial moisture content, bulk density and sand-silt-clay contents. Another group of these factors are related to the drip irrigation system, e.g. the emitter flow rate, the volume of applied water, the irrigation application mode (e.g. continuous or pulse), the emitter position (e.g. surface or subsurface), and the elapsed time (Mohammad beige et 
al., 2016; Karimi et al., 2013). Accordingly, an empirical nonlinear regression model is proposed here to estimate the dimensions of moisture redistribution. Using the data obtained from the laboratory experiments and conducting the nonlinear regression analysis using Microsoft ExcelSolver tool 2010 (as discussed by Al-Ogaidi et al., 2016), the coefficients of the following were inferred. In this research, 108, 126, and 111 patterns were used for modeling the $D_{r e}\left(D_{r e}=2 R_{r e}\right), V_{r e}$ and $A_{r e}$ for the pulse surface drip irrigation system, respectively. The general forms of the proposed methods are presented in the Eqs. (1-3). These expressions were developed on the basis of the previously published literature (e.g. Al-ogaidi et al., 2016).

152

$$
\begin{aligned}
& D_{\text {re }}=a^{a_{1}} Q^{a_{2}} q^{a_{3}} k_{s}^{a_{4}} S^{a_{5}} S^{a_{6}} C^{a_{7}} \rho_{b}{ }^{a_{8}} \theta_{i}{ }^{a_{9}}\left(\frac{T_{\text {on }}}{T_{\text {tot }}}\right)^{a_{10}} \\
& V_{\text {re }}=b^{b_{1}} Q^{b_{2}} q^{b_{3}} k_{s}^{b_{4}} S^{b_{5}} S^{b_{6}} C^{b_{7}} \rho_{b} b_{8} \theta_{i} b_{9}\left(\frac{T_{\text {on }}}{T_{\text {tot }}}\right)^{b_{10}} \\
& A_{\text {re }}=c^{c_{1}} Q^{c_{2}} q^{c_{3}} k_{s}{ }^{c_{4}} S^{c_{5}} S^{c_{6}} C^{c_{7}} \rho_{b}{ }^{c_{8}} \theta_{i}{ }^{c_{9}}\left(\frac{T_{\text {on }}}{T_{\text {tot }}}\right)^{c_{10}}
\end{aligned}
$$

Further, the number of patterns used for modeling the $D_{r e}, \mathrm{~V}_{\text {re }}, A_{r e}, A_{\text {re-up }}$ for the pulse subsurface models for this state are as follows.

$$
\begin{aligned}
& D_{\text {re }}=d t^{d_{1}} Q^{d_{2}} q^{d_{3}} k_{s}^{d_{4}} S^{d_{5}} S^{d_{6}} C^{d_{7}} \rho_{b}{ }^{d_{8}} \theta_{i}{ }^{d_{9}} Z^{d_{10}}\left(\frac{T_{\text {on }}}{T_{\text {tot }}}\right)^{d_{11}} \\
& V_{\text {re }}=\text { et }^{\mathrm{e}_{1}} Q^{\mathrm{e}_{2}} \mathrm{q}^{\mathrm{e}_{3}} \mathrm{k}_{\mathrm{s}}{ }^{\mathrm{e}_{4}} \mathrm{~S}^{\mathrm{e}_{5}} \mathrm{Si}^{\mathrm{e}_{6}} \mathrm{C}^{\mathrm{e}_{7}} \rho_{\mathrm{b}}{ }^{\mathrm{e}_{8}} \theta_{\mathrm{i}}{ }^{\mathrm{e}_{9}} \mathrm{Z}^{\mathrm{e}_{10}}\left(\frac{\mathrm{T}_{\mathrm{on}}}{\mathrm{T}_{\mathrm{tot}}}\right)^{\mathrm{e}_{11}} \\
& A_{r e}=f^{f_{1}} Q^{f_{2}} q^{f_{3}} k_{s}^{f_{4}} S^{f_{5}} S^{f_{6}} C^{f_{7}} \rho_{b}{ }^{f_{8}} \theta_{i} f^{f_{9}} Z^{f_{10}}\left(\frac{T_{\text {on }}}{T_{\text {tot }}}\right)^{f_{11}} \\
& A_{\text {re-up }}=g^{g_{1}} Q^{g_{2}} q^{g_{3}} k_{s}^{g_{4}} S^{g_{5}} S^{g_{6}} C^{g_{7}} \rho_{b}{ }^{g_{8}} \theta_{i}{ }^{g_{9}} Z^{g_{10}}\left(\frac{T_{\text {on }}}{T_{\text {tot }}}\right)^{g_{11}}
\end{aligned}
$$


In these equations, $\mathrm{D}_{\text {re: }}$ : the diameter of horizontal redistribution $\left(D_{r e}=2 R_{r e}\right) ; \mathrm{V}_{\text {re }}$ and $\mathrm{V}_{\text {re-up: }}$ : downward and upward vertical redistribution, respectively (all in $\mathrm{cm}$ ); $A_{r e}$ : lower side of the emitter wetted area after cut-offing the irrigation process and $A_{\text {re-up }}$ : the upper side of the emitter wetted area after cut-offing the irrigation process (both in $\mathrm{cm}^{2}$ ). In addition, $t$ is the elapsed time after cut-offing the irrigation process $(\min ), q$ denotes the emitter discharge (lit/hr ), $Q$ stands for the volume of applied water during irrigation (lit), $K_{S}$ shows the soil saturated hydraulic conductivity $(\mathrm{cm} / \mathrm{hr}), S$, $S i$, and $C$ are, respectively, the sand, silt, and clay content $(\%), \rho_{b}$ shows the soil bulk density

to the entire cycle time in the pulse irrigation system.

\subsection{Statistical criteria}

The results of the proposed models were assessed using four statistical evaluation indices, namely, the mean absolute error (MAE), the root mean square error (RMSE), the Nash-Sutcliffe coefficient (NS) and the determination coefficient $\left(\mathrm{R}^{2}\right)$ which was computed using the following equations:

$$
\begin{aligned}
& R^{2}=\left[\frac{\sum_{i=1}^{n}\left(x_{i}^{\text {target }}-\overline{x^{\text {target }}}\right)\left(x_{i}^{\text {model }}-\overline{x^{\text {model }}}\right)}{\sqrt{\sum_{i=1}^{n}\left(x_{i}^{t \arg e t}-\overline{x^{\text {target }}}\right)^{2} \sum_{i=1}^{n}\left(x_{i}^{\text {model }}-\overline{x^{\text {model }}}\right)^{2}}}\right]^{2} \\
& R M S E=\sqrt{\frac{1}{n} \sum_{i=1}^{n}\left(x_{i}^{\text {model }}-x_{i}^{t \arg e t}\right)^{2}} \\
& M A E=\frac{1}{n}\left|\sum_{i=1}^{n}\left(x_{i}^{\bmod e l}-x_{i}^{t a r g e t}\right)\right|
\end{aligned}
$$




$$
N S=1-\frac{\sum_{i=1}^{n}\left(x_{i}^{\bmod e l}-x_{i}^{t \arg e t}\right)^{2}}{\sum_{i=1}^{n}\left(x_{i}^{t \arg e t}-\overline{x^{t \arg e t}}\right)^{2}}
$$

Where $x^{\text {model }}$ and $x^{t \text { arget }}$ indicate the estimated and observed data, respectively. $\overline{x^{\text {model }}}$ and $\overline{x^{t \text { arg } e t}}$ are the mean values of the predicted and observed parameter, respectively. $\mathrm{n}$ is the total number of data.

\section{Results and discussions}

\subsection{Evaluation of the moisture redistribution}

Figure 3 shows the moisture redistribution amounts in both horizontal and vertical directions for redistribution after irrigation cut-off time has considerable values in both the directions. The horizontal and vertical redistribution showed higher values for the light texture soils (LT in the figure), except for pulse irrigation mode with the flow rate of 6 lit/hr. The higher redistribution values for the light-textured soils might be linked to the water percolation into the soil depths, where the outflow from the emitters penetrates and accumulates in sandy (light-textured) soils due to the higher permeability of these textures. In case of the medium/heavy-textured soils, however, water flow tends to generally move across the soil surface, so lower amount of water accumulates in the soil (Karimi et al., 2013; Mohammad beige et al., 2016).

Further, by increasing the flow rate, the horizontal and vertical redistribution values were also partially increased. This could be anticipated, because at higher emitter flow rates, more water volume accumulates in the soil and gradually redistributed after cut-offing the irrigation process. The results of Fig. 3 showed that the horizontal redistribution in the continuous irrigation system is much higher than the pulse irrigation system, which might be linked to the lower water penetration opportunity in the continuous irrigation system that facilitate the horizontal redistribution of water, as discussed by Mohammadbeigi et al. (2016). Fig. 4, shows the moisture redistribution values for 
the subsurface irrigation system. Similar to the surface irrigation system, as could be anticipated, the highest and lowest redistribution values are corresponded to the light-textured (LT) and heavytextured (HT) soils, respectively. The results of Fig. 4 also indicated that with increasing the flow rate, the horizontal redistribution in the continuous subsurface irrigation system partly increases, confirming the conclusions obtained by Karimi et al. (2013). The reason behind this might be the fact that by increasing the emitter discharge, the soil capability for transmitting the water during irrigation is decreased, so the water collected around the emitter moves horizontally. Tables 2- 3 present the percentage of the wetted dimensions and area of moisture redistribution for the continuous and pulse surface/subsurface irrigation modes. For all the treatments, the ratios of the horizontal redistribution to horizontal distribution $\left(\frac{D_{r e}}{D}\right)$, vertical redistribution to vertical distribution $\left(\frac{V_{r e}}{V}\right)$, upward vertical redistribution to vertical distribution $\left(\frac{V_{r e-u p}}{V}\right)$, emitter lower wetted area to total wetted area $\left(\frac{A_{r e}}{A_{\text {irr }}}\right)$, and the emitter upper wetted area to total wetted area $\left(\frac{A_{r e-u p}}{A_{\text {irr }}}\right)$, were calculated at the time of irrigation. These results indicated that most of the wetted pattern of wetting bulb is related to moisture redistribution. Further, the results indicated that a significant percentage of the wetted areas at the lower and upper sides of the emitters are corresponded to the redistribution of moisture after cut-offing the irrigation. The results showed that the ratio of $\frac{A_{r e}}{A_{\text {irr }}}$ for the studied treatments varied between $0.197-0.503,0.159-0.505,0.155-0.526$, and $0.141-0.333$ for continuous and pulse irrigation (the values of 30-30, 40-20, and 20-40), respectively. Moreover, the $\frac{A_{r e-u p}}{A_{\text {irr }}}$ values varied between $0.043-0.149,0.023-0.085,0.03-0.126$, and $0.037-0.111$ for the mentioned treatments, respectively. These values indicated that significant amount of moisture bulb pattern is formed by moisture redistribution that should be considered in designing the irrigation systems.

Table4 summarizes the total dimensions attained by the wet patterns for both the continuous and pulse irrigation systems. The horizontal dimension can be used for determining the distance between the emitters and laterals, while the vertical values might provide information for selecting the best 
installation depth of the emitters in subsurface systems. From the table, the maximum total horizontal distribution was observed in sandy soils (coarse texture) for the continuous irrigation system (except: DI-CT- $\mathrm{q}_{3}$ and SDI-30-CT- $\mathrm{q}_{2}$ ). This might be due to transferring a vast amount of water into the soil profile through continuous irrigation as well as the ease of horizontal/vertical water movement in sandy soils. Similar outcomes might be stated for vertical distribution values, 30-30.

which were higher in coarse texture than the fine and moisture ones for both the surface and subsurface continuous/pulse irrigation systems. Finally, the highest amount of total vertical distribution and wetted area in pulse subsurface irrigation system corresponded to the pulse ratio of

\subsection{Analysis of the suggested models}

The obtained coefficients of the proposed non-linear regression models are presented in Table5. By analyzing the values presented in this table, some remarks can be highlighted. First, the statistical parameters clearly demonstrated the good ability of the proposed models in estimating redistribution values for both the surface and sub-surface pulse irrigation systems. Based on the statistical indices, the proposed models has showed good ability for simulating pattern of wetting redistribution in surface systems (equations 1-3) if compared with the previous literature (e.g. Al-Ogaidi et al., 2016). In addition, the statistical indices corresponded to the subsurface irrigation systems indicated that the proposed models have a good performance in estimating the redistribution dimensions when compared by previous studies (e.g. Malek and Peters, 2011).

\subsubsection{Pulse surface drip irrigation}

The outcomes of the statistical parameters of Table 6 show that the proposed models predict the horizontal and vertical redistribution in surface drip irrigation (SDI) system with acceptable accuracy. Further, in Fig. 5, comparing the measured and simulated values indicated that the 
proposed model presented good ability in simulating the horizontal redistribution in the SDI system for all adopted pulse ratios (20-40, 30-30, 40-20) and soil textures (MT, LT, HT). In addition, according to Table 6 , these models estimated the vertical redistribution in the surface irrigation system with suitable performance accuracy. The MAE, RMSE and NS values for the studied treatments vary between $0.26-0.59,0.3-0.79 \mathrm{~cm}$ and $0.74-0.97$, respectively. Table 7 sums up the

\subsubsection{Pulse subsurface drip irrigation}

The statistical indices for the proposed models of the pulse subsurface irrigation system have been vertical redistribution in the SDI systems with acceptable performance. The MAE, RMSE and NS values varied between $0.34-0.72 \mathrm{~cm}, 0.37-0.83 \mathrm{~cm}$ and $0.77-0.96$, respectively for the horizontal redistribution values, while they varied, respectively, between $0.13-0.42,0.17-0.48 \mathrm{~cm}$ and $0.65-0.96$ for vertical redistribution simulation. Nonetheless, comparing the measured and simulated redistribution values in Figure 6 showed that the proposed models have acceptable performances. . Table9 lists the statistical indices of the models for simulating the wetted area of the emitters of SDI system after cut-offing the irrigation process. According to the table, it is proved that these models estimated the emitters' lower wetted area with the MAE, RMSE and NS values varying between the 0.0014-0.0066 $\mathrm{m} 2,0.0021-0.0077 \mathrm{~m}^{2}$ and $0.79-0.98$, respectively. The values of these indices varied 
between $\quad 0.0008-0.0019 \mathrm{~m} 2,0.001-0.0022 \mathrm{~m}^{2}$ and $0.72-0.96$, respectively for upper wetted area modeling in SDI system. Similar partial conclusions might be obtained by assessing the scatterplots presented in Figure 6, confirming the outcomes reported by Karimi et al. (2015a).

\section{Conclusion}

Due to the importance of the moisture redistribution in soil, a set of experiments were carried out for surface and subsurface drip irrigation systems using three various outflow rates (e.g., 2, 4, and 6 lit/hr) and three different soil types (clay, sandy-clay-loam, and sandy-loam) for both continuous and pulse irrigation systems (i.e., with the ratios of 20-40, 30-30, 40-20). Some non-linear regression based equations were also proposed for estimating the moisture redistribution dimensions under the mentioned irrigation systems. The outcomes of the present research revealed that:

2. The horizontal and vertical redistribution showed higher values for the light texture soils.

3. The horizontal redistribution in the continuous irrigation system was higher than the pulse irrigation system.

4. The proposed non-linear regression models estimated the horizontal/vertical redistribution and lower/ upper wetted area in surface/subsurface pulse drip irrigation system with acceptable accuracy.

5. The utilization of these techniques for design purposes can be useful in determining the exact space between laterals and emitters as well as the appropriate depth of emitters to reduce water losses via surface runoff and deep percolation.

\section{Reference}


Al-Ogaidi, A.A.M., Wayayok A., Rowshona, M.K. and Abdullah, A.F. 2016. Wetting patterns estimation under drip irrigation systems using an enhanced empirical model. Agric. Water Manage. 176: 203-213.

Amin, M.S.M., Ekhmaj, A.I.M., 2006. DIPAC- drip irrigation water distribution pattern calculator. In: 7th Int Micro Irrigation Congress PWTC, Kuala Lumpur, Malaysia, pp. 503-513.

Cook, F.J., Thorburn, P.J., Fitch, P., Bristow, K.L., 2003. WetUp: a software tool to display approximate wetting patterns from drippers. Irrig. Sci. 22: 129-134.

Elmaloglou, S., and Diamantopoulos, E. 2009. Effects of hysteresis on redistribution of soil moisture and deep percolation at continuous and pulse drip irrigation. Agric. Water Manag. 96: 533-538.

Elmaloglou, S., and Diamantopoulos, E. 2010. Soil water dynamics under surface trickle irrigation as affected by soil hydraulic properties, discharge rate, dripper spacing and irrigation duration. Irrig. Drain. 263: 254263.

Golestani Kermani, S., Sayari, S., Kisi, O., Zounemat-Kermani, M., 2019. Comparing data driven models versus numerical models in simulation of waterfront advance in furrow irrigation. Irrig. Sci. 37(5), 547-560.

Hammami, M., Zayani, K.2016. An analytical approach to predict the moistened bulb volume beneath a surface point source. Agric. Water Manag. 166: 123-129.

Kandelous, M.M., Simunek, J., 2010a. Comparison of numerical, analytical and empirical models to estimate wetting pattern for surface and subsurface drip irrigation. Irrig. Sci. 28: 435-444.

Kandelous, M.M., Simunek, J., 2010b. Numerical simulations of water movement in a subsurface drip irrigation system under field and laboratory conditions using HYDRUS-2D. Agric. Water Manag. 97: 1070-1076.

Karimi, B., Mirzaei, F., and Sohrabi, T.2013. Evaluation of moisture front redistribution in surface and subsurface drip irrigation systems. Iranian J. Water and Soil Sci. 23(3): 183-192.

Karimi, B., Mirzaei, F. and Sohrabi, T. 2015a. Developing Equations to Estimate Wetted Area Pattern for Surface and Subsurface Drip Irrigation Systems by Dimensional Analysis. Iranian J. Soil and Water Sci. 25(3): 241:252.

Karimi, B., Sohrabi, T., Mirzaei, F., and Ababaei, B.2015b. Developing equations to predict the pattern of soils moisture redistribution in surface and subsurface drip irrigation systems using dimension analysis. Iranian J. Water and Soil Conser. 21(6): 223-237. 
Karimi, B., Mohammadi, P., Sanikhani, H., Salih, S.Q. and Yaseen, Z.M., 2020. Modeling wetted areas of moisture bulb for drip irrigation systems: An enhanced empirical model and artificial neural network. Computers and Electronics in Agriculture, 178, p.105767.

Karmeli, D., Peri, G., 1974. Basic principles of pulse irrigation. J. Irrig. and Drain. Division. ASCE 100 (3): $309-319$.

Li, J., Zhang, J., Ren L. 2003. Water and nitrogen distribution as affected by fertigation of ammonium nitrate from a point source. Irrig. Sci. 22(1):19-30.

Liu, Zhigang., Xu, Qinchao. 2018. Wetting patterns estimation in cultivation substrates under drip irrigation. Desa. and water Treat. 112 (2018): 319-324.

Malek, K., Peters, R.T., 2011. Wetting pattern models for drip irrigation: new empirical model. J. Irrig. Drain. Eng. 137, 530-536.

Mohammadbeigi, A., Mirzaei, F., and Ashraf, N.2016. Evaluation and comparing of redistribution of moisture in drip irrigation by pulsed flow and continuous flow. Iranian J. of Soil and Water Rese. 47(3): 467-473.

Mohammadbeigi, A., Mirzaei, F., and Ashraf, N.2017. Simulation of soil moisture distribution under drip irrigation pulsed and continuous in dimensional analysis method. Iranian J. of Water and Soil Conser. 23(6): 163-180.

Mostaghimi, S., Mitchell, J.K., 1983. Pulsed trickling effect on soil moisture distribution. Water Reso. Bull. 19 (4): 605-612.

Qiaosheng, Sh., Zouxin L., Zhenying, W., and Hayjung L. 2007. Simulation of the soil wetting shape under porous pipe sub-Irrigation using dimensional analysis. J. Irrig. Drain. Eng. 125: 389- 398.

Schaap, M.G., Leij, F.J., van Genuchten, M.T., 2001. Rosetta: a computer program for estimating soil hydraulic parameters with hierarchical pedotransfer functions. J. Hydrol. 251: 163-176. 
342 Schwartzman M., Zur B.1986. Emitter Spacing and Geometry of Wetted Soil Volume. J. Irrig. Drain Eng. 112(3): 242-253.

344 Shiri, J., Karimi, B., Karimi, N., Kazemi, M.H. and Karimi, S., 2020. Simulating wetting front dimensions of drip irrigation systems: Multi criteria assessment of soft computing models. J. Hydrol., 585: p.124792. subsurface drip irrigation from line source. Agric. Water Manag. 83:130-134. irrigation. Irrig. Sci. 29: 331-339. 


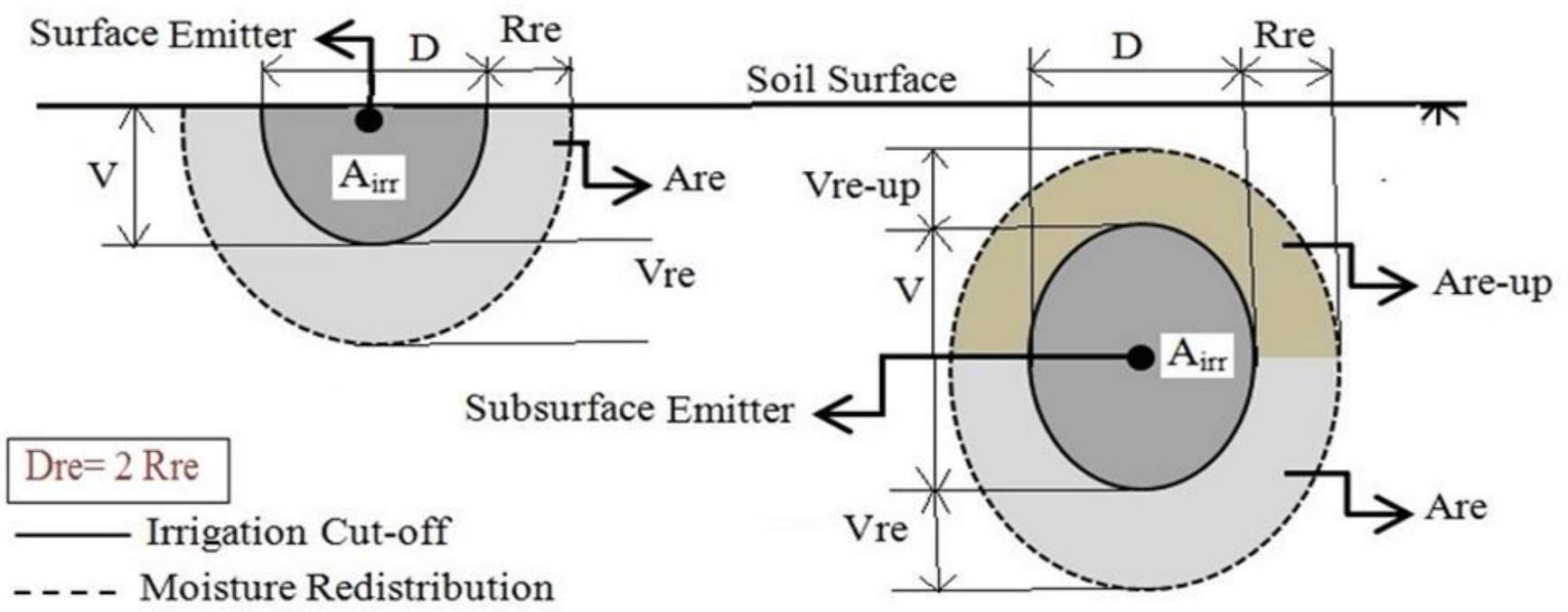

Figure 1

Schematic representation of the moisture redistribution in surface and subsurface drip irrigation systems (D: horizontal distribution during irrigation; V: vertical distribution during irrigation; Airr: wetted area around emitter during irrigation; Rre: horizontal redistribution; Vre and Vre-up: downward and upward vertical redistribution, respectively; Are and Are-up: lower and upper wetted area after cut-offing the irrigation process, respectively)

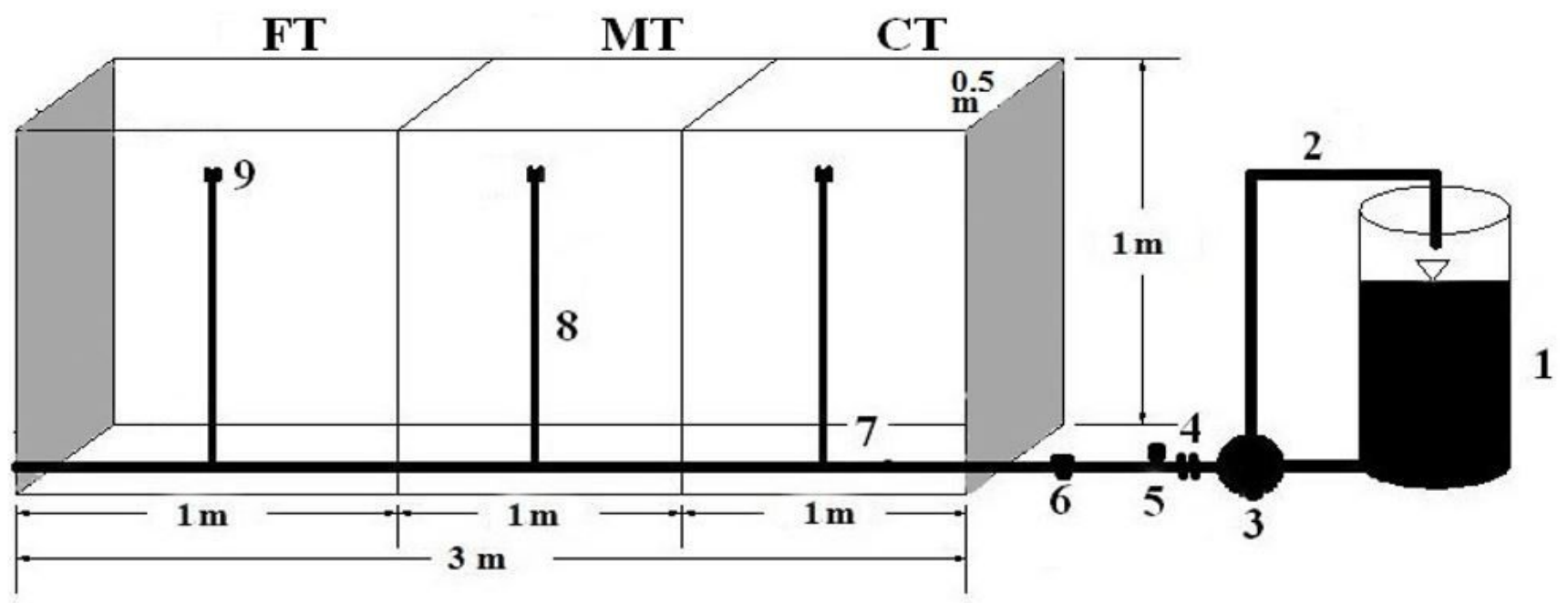

Figure 2 
Schematic descriptions of the experimental device. 1-Reservoir; 2- By-pass assembly; 3- Pump; 4-Valve; 5Gage; 6- Filter; 7- Main pipe; 8- Sub-main pipe; 9- Off-on valve.
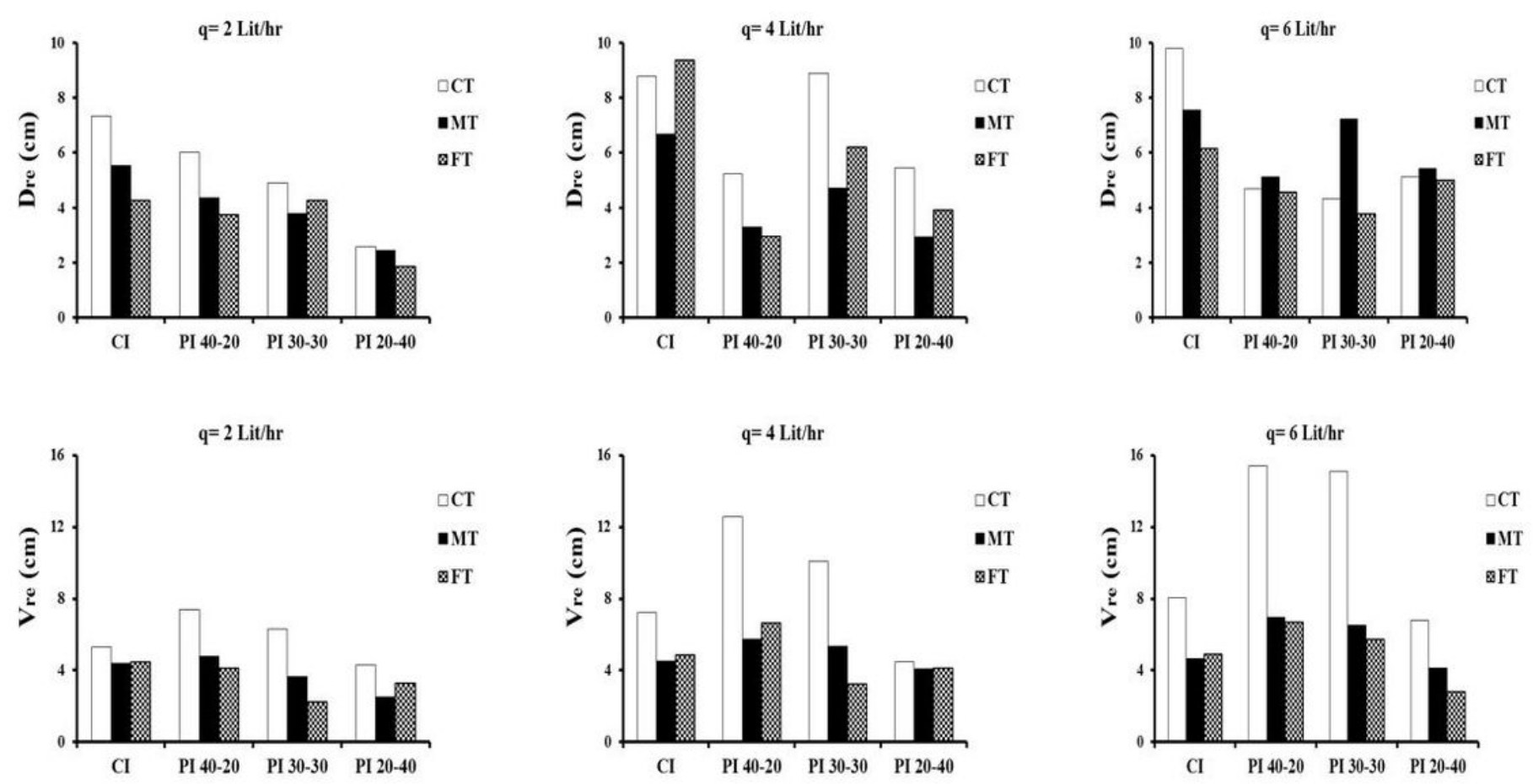

Figure 3

Values of horizontal and vertical moisture redistribution for surface drip irrigation (Cl: Continuous irrigation; PI: Pulse irrigation; CT: coarse texture; MT: medium texture; FT: fine texture) 

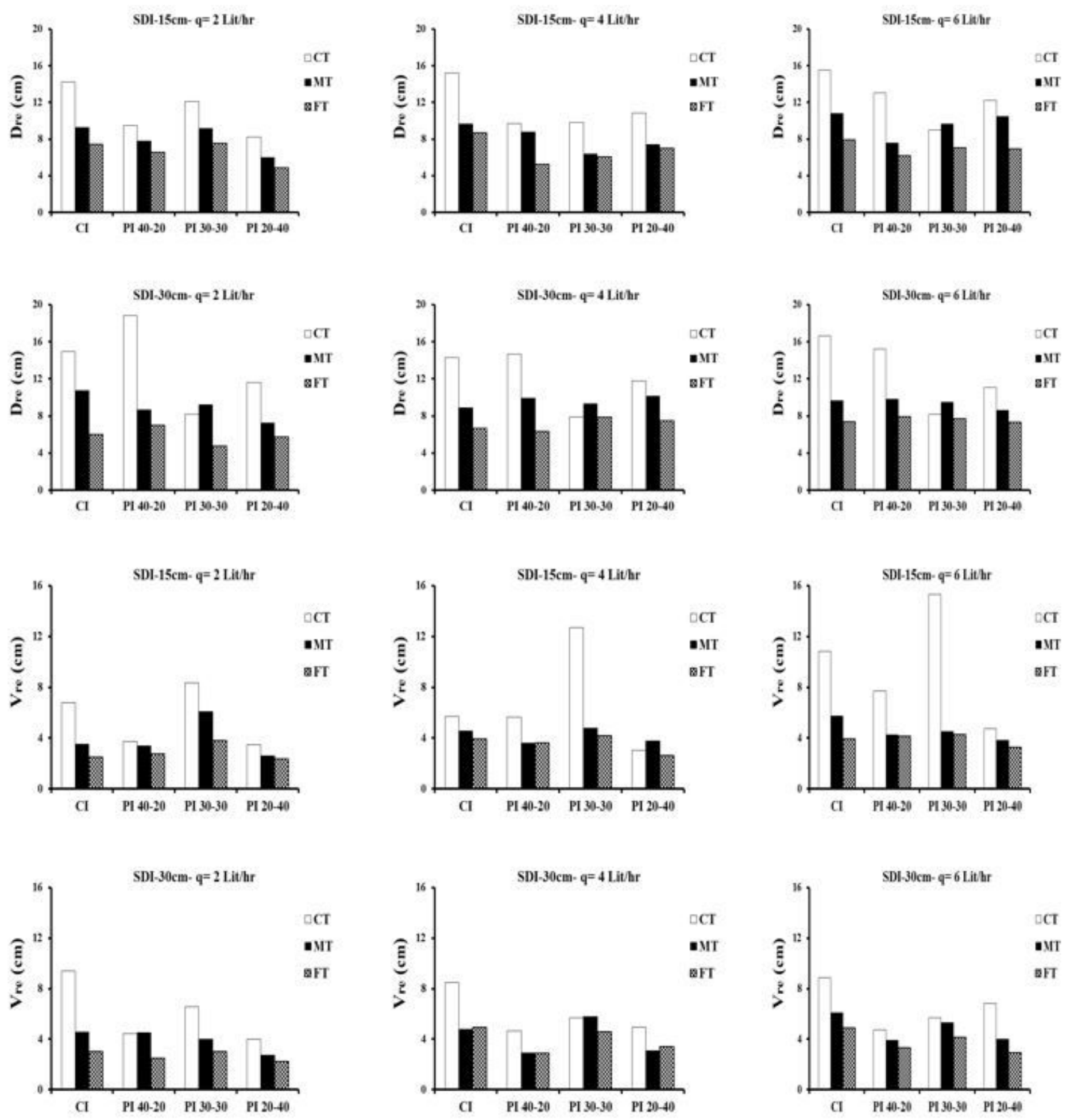

Figure 4

Values of horizontal and vertical moisture redistribution for subsurface drip irrigation (Cl: Continuous irrigation; PI: Pulse irrigation; CT: coarse texture; MT: medium texture; FT: fine texture) 

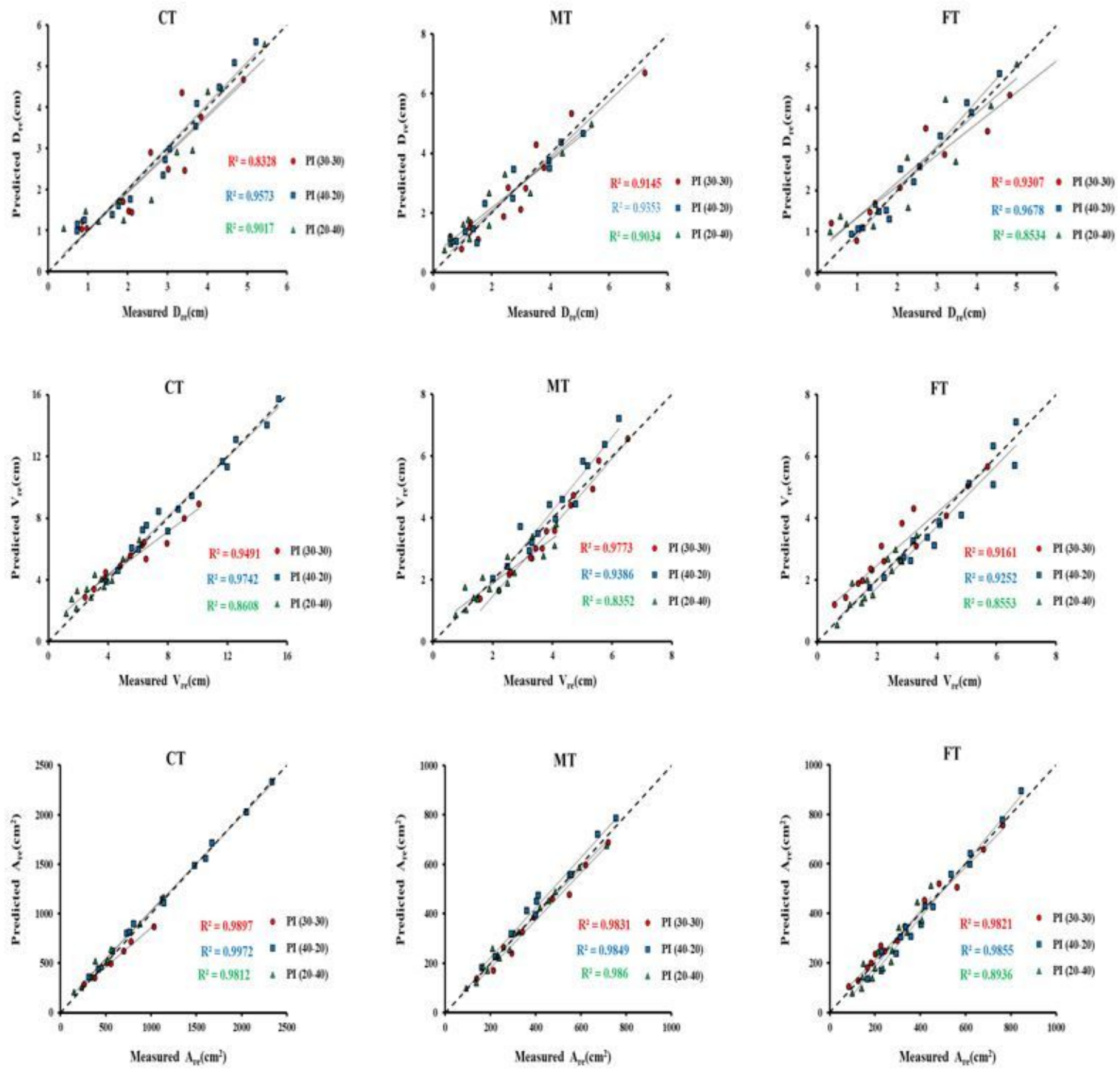

Figure 5

Comparison of measured and simulated values of redistribution (Dre, Vre and Are) for pulse surface drip irrigation (CT: coarse texture; MT: medium texture; FT: fine texture) 

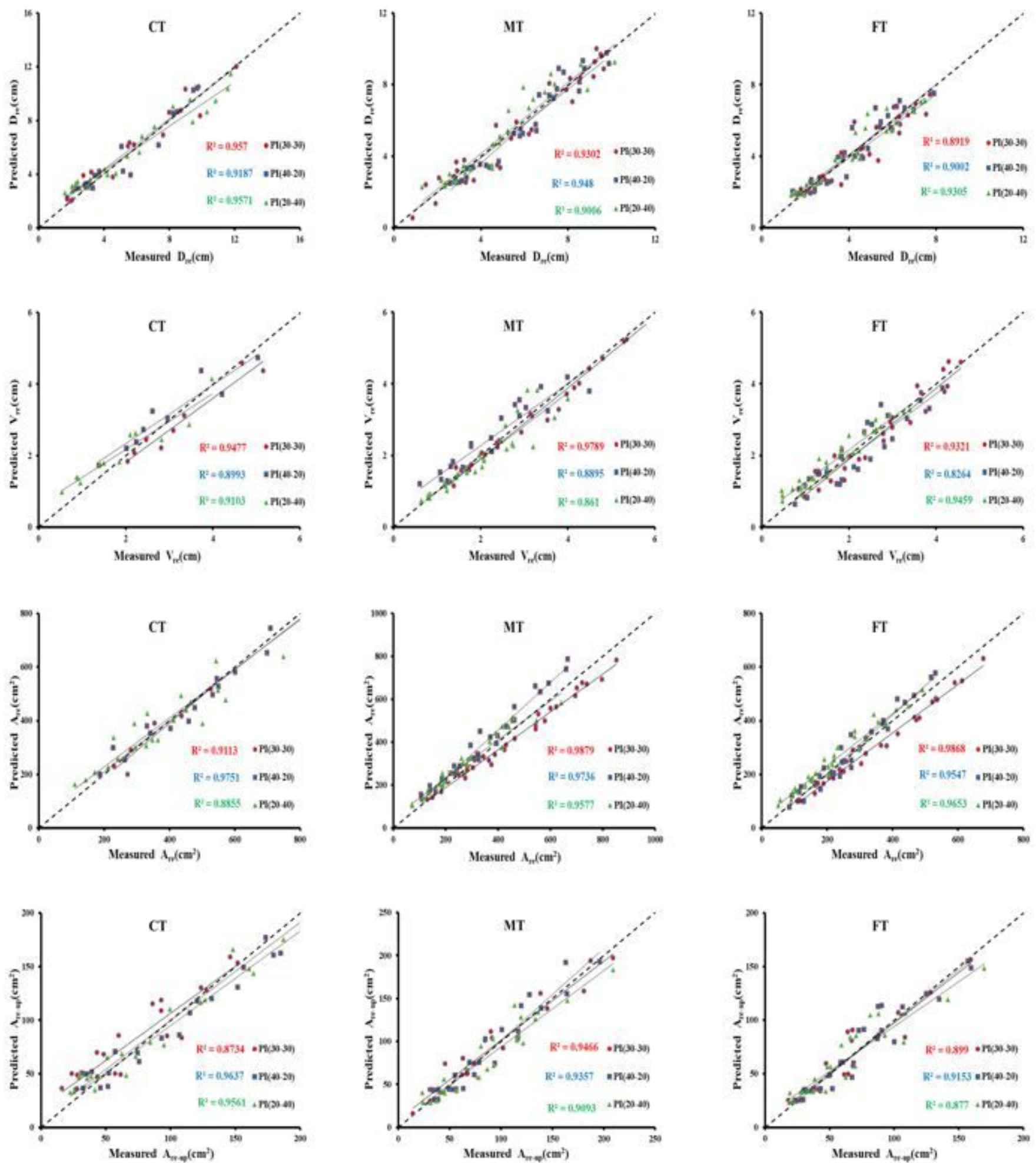

Figure 6

Comparison of measured and simulated values of redistribution (Dre, Vre, Are, and Are-up) for pulse subsurface drip irrigation 
This is a list of supplementary files associated with this preprint. Click to download.

- Table.docx 